\title{
Cell nuclei detection using globally optimal active contours with shape prior
}

\author{
Jonas De Vylder ${ }^{1 *}$, Jan Aelterman ${ }^{1}$, Mado Vandewoestyne ${ }^{2}$, Trees Lepez ${ }^{2}$, Dieter \\ Deforce $^{2}$, and Wilfried Philips ${ }^{1}$ \\ ${ }^{1}$ Department of Telecommunications and Information Processing, \\ IBBT - Image Processing and Interpretation, Ghent University, Ghent, Belgium \\ ${ }^{2}$ Laboratory of Pharmaceutical Biotechnology, Faculty of Pharmaceutical Sciences, \\ Ghent University, Ghent, Belgium \\ jonas.devylderetelin.ugent.be \\ http://telin.ugent.be/ jdvylder
}

\begin{abstract}
Cell nuclei detection in fluorescent microscopic images is an important and time consuming task for a wide range of biological applications. Blur, clutter, bleed through and partial occlusion of nuclei make this a challenging task for automated detection of individual nuclei using image analysis. This paper proposes a novel and robust detection method based on the active contour framework. The method exploits prior knowledge of the nucleus shape in order to better detect individual nuclei. The method is formulated as the optimization of a convex energy function. The proposed method shows accurate detection results even for clusters of nuclei where state of the art methods fail.
\end{abstract}

\section{Introduction}

Cell nuclei are one of the most studied objects in microscopic biology. This is because they are easily visualized independent of the type of cells, typically using a fluorescent staining, while containing relevant biological information for a wide range of applications, e.g. cell division in tumours, root growth in plants, full embryonic development, etc. [1,2].

Due to the biological importance of cell nuclei, several automated detection methods have been proposed in the past. These methods can generally be categorized in two groups: edge based and intensity based. The first group starts by detecting edges, both binary and continuous edge maps have been used, and tries to fit a specific shape model to them [3-6]. The robustness of these methods strongly depends on the output of the edge detector, which is not always sufficient to detect individual nuclei in case of clustered nuclei. The second group first segments the nuclei from the background, this is typically done based on intensity, e.g. by some sort of automatic thresholding [7, 8]. For isolated nuclei this is rather straightforward, but for touching nuclei this requires an extra step to detect each individual nucleus. This is mainly done by assuming that the detected segments should have a convex shape [2,9-11]. These methods are non optimal since both steps are independent: the second step can only use the result of the first step, instead of all information available, e.g. the complete image. Incorrectly segmented pixels can have a big influence on the individual detection. 
In contrast too state of the art methods, this paper proposes an intensity based segmentation method which tackles the individual nuclei detection in a single step. The decision of segmenting a pixel as foreground is based both on intensity and on the likelihood that a nucleus is located at that location. This combined approach will result in more accurate nuclei detection as will be shown in the results section. This is achieved using the active contour framework. The proposed method has a convex energy function, thus is invariant of the initialization $[12,13]$. This paper is arranged as follows. The next section provides a brief description of convex energy active contours. In section 4 our proposed algorithm is presented, while section 5 elaborates on the optimization of our proposed method. Section 6 shows the results of our technique and the convergence of our method is studied. Section 7 recapitulates and concludes.

\section{Notations and Definitions}

In the remaining of this paper we will use specific notations. To make sure all notations are clear, we briefly summarize the notations and symbols used in this work.

We will refer to an image, $F$ in its vector notation, i.e. $\mathbf{f}(i * m+j)=F(i, j)$, where $m \times n$ is the dimension of the image. In a similar way we will represent the contour in vector format, $\mathbf{u}$. If a pixel $(i, j)$ is part of the segment, it will have a value above a certain threshold, all background pixels will have a value lower than the given threshold. Note that this is similar to level-sets. The way these contours are optimized however is different than with classical level-set active contours, as is explained in the next section. We will use the gradient image operators in combination with this vector notation, however the semantics of this operator remains the same as if it was used with the classical matrix notation:

$$
\nabla(\mathbf{f}(i * m+j))=(F(i+1, j)-F(i, j), F(i, j+1)-F(i, j))
$$

Further we will use the following inner product and norm notations:

$$
\begin{aligned}
\langle\mathbf{f}, \mathbf{g}\rangle & =\sum_{i=1}^{m n} f(i) g(i) \\
\|\mathbf{f}\|_{0} & =\sum_{i=1}^{m n} 1-\delta_{0, f(i)} \\
|\mathbf{f}| & =\sum_{i=1}^{m n}|f(i)| \\
\|\mathbf{f}\|_{2} & =\sqrt{\sum_{i=1}^{m n} f(i)^{2}}
\end{aligned}
$$

Where the weights $g(i) \geq 0$ and $\delta_{i, j}$ represents the Kronecker delta, which is equal to one if $i$ and $j$ are equal and is zero in all other cases. The $l 0$ norm, i.e. $\|\mathbf{f}\|_{0}$, counts the non-zero elements of the vector. Note that the $l 0$ norm is not a real norm in the mathematical sense, since the triangle inequality does not hold. 


\section{Convex Energy Active Contours}

In [12] an active contour model was proposed which has global minimisers. This active contour is calculated by minimizing the following convex energy:

$$
E[\mathbf{u}]=|\nabla \mathbf{u}|+\beta\langle\mathbf{u}, \mathbf{r}\rangle
$$

with

$$
\mathbf{r}=\left(\mu_{f}-\mathbf{f}\right)^{2}-\left(\mu_{b}-\mathbf{f}\right)^{2}
$$

where $\mu_{f}, \mu_{b}$ are respectively the expected foreground and background intensity and $\beta$ is a weighting parameter tuning the influence of the data-fit in relation to the total variation regularization. Note that this energy is convex, only if $\mu_{f}$ and $\mu_{b}$ are constant. The expected intensities $\mu_{f}, \mu_{b}$ can be calculated from a training dataset. Chan et al. [12] proved that $\mathbf{u}$ is well-defined as the solution of a convex energy function over a convex domain if $\mathbf{u} \in[0,1]^{m n}$, i.e.

$$
\hat{\mathbf{u}}=\underset{\mathbf{u} \in[0,1]^{m n}}{\arg \min }|\nabla \mathbf{u}|+\beta\langle\mathbf{u}, \mathbf{r}\rangle
$$

Furthermore, the steady state of the gradient flow corresponding to this energy function coincides with the steady state of the gradient flow of the original active contours without edges (ACWE) $[12,14]$, i.e. an optimum of this convex energy is also an optimum of the original ACWE energy function. Note that $\hat{\mathbf{u}}[i]$ can have any value between 0 and 1, thus the found active contour does not have to represent a crisp segmentation. A binary segmentation result can be given by thresholding $\hat{\mathbf{u}}$, i.e.

$$
\Phi_{\alpha}(\hat{\mathbf{u}}[i])= \begin{cases}1 & \text { if } \hat{\mathbf{u}}[i]>\alpha \\ 0 & \text { otherwise }\end{cases}
$$

for some $\alpha \in[0,1]$. In [13] it is shown that $\Phi_{\alpha}(\hat{\mathbf{u}})$ itself is a global minimizer for the energy in eq. (2) and by extension for the energy function of the ACWE model.

\section{Active Contours with Sparse Shape Prior}

The energy function in eq. (2) tries to remove noisy segmentation pixels by regularizing the energy function using total variation. This regularization is useful if pixels are incorrectly classified, i.e. background pixels detected as foreground or vice versa, due to noise in the image. In microscopic images however, incorrectly detected nuclei are often caused by clutter in the image, e.g. dead cells or bleed-through from other fluorescent channels. This is not solved using total variation since these incorrectly detected nuclei correspond to natural objects. Therefore, we propose a regularization term that exploits the regular shape of cell nuclei, penalizing segments with strongly deviate from the expected shape. In this work, we model a nucleus as a disk. Thus, given a predefined radius, $r$, and location, $(\mathrm{x}, \mathrm{y})$, we can calculate the ideal $\mathbf{u}$, i.e. a binary image where the pixels within a distance $\mathrm{r}$ of $(\mathrm{x}, \mathrm{y})$ are equal to one and all other pixels are equal to zero. For a discrete range of nuclei diameters it is possible to enumerate all possible u's, this 
is of course under the assumption that there is only one nucleus in the image. We will refer to each of these possible u's as words. In most applications however the image does contain multiple nuclei, where it is unknown how many nuclei are present, this requires a superposition of multiple words to represent $\mathbf{u}$. The optimal active contour can be represented using a minimal number of words, i.e. one word per nucleus. This representation of the active contour in terms of a superposition of words can be used as a new regularization term:

$$
\hat{\mathbf{u}}, \hat{\mathbf{t}}=\underset{\mathbf{t}, \mathbf{u} \in[0,1]^{m n}}{\arg \min }\|\mathbf{t}\|_{0}+\beta\langle\mathbf{u}, \mathbf{r}\rangle \quad \text { such that } \quad \mathbf{u}=D \mathbf{t}
$$

where $D$ a matrix of dimension $m n r \times m n$, representing a dictionary of words, with $m n$ corresponding to the number of pixels, and $r$ corresponding to the number of possible diameters of a nucleus. Thus we can represent this dictionary as

$D=\left[\mathbf{d}_{\mathbf{a}, \mathbf{1}} \mathbf{d}_{\mathbf{a}, \mathbf{2}} \mathbf{d}_{\mathbf{a}, \mathbf{3}} \ldots \mathbf{d}_{\mathbf{a}+\mathbf{1}, \mathbf{1}} \mathbf{d}_{\mathbf{a}+\mathbf{1}, \mathbf{2}} \mathbf{d}_{\mathbf{a}+\mathbf{1}, \mathbf{3}} \ldots \mathbf{d}_{\mathbf{b}, \mathbf{n}}\right]$ with $\mathbf{d}_{\mathbf{i}, \mathbf{j}}$ the vector form of a word, i.e. a binary representation of a disk with radius $i \in[a, b]$ and centroid at pixel $j$. Thus, $\mathbf{t}$ represents a weighting vector for the words. The energy term minimized by $\mathbf{u}$ is based on a $l_{0}$ norm which is non-convex. Fortunately the $l_{1}$ norm can be used as a good approximation instead [15]. This new prior results in the following active contour:

$$
\hat{\mathbf{u}}, \hat{\mathbf{t}}=\underset{\mathbf{t}, \mathbf{u} \in[0,1]^{m n}}{\arg \min }|\mathbf{t}|+\beta\langle\mathbf{u}, \mathbf{r}\rangle \quad \text { such that } \quad \mathbf{u}=D \mathbf{t}
$$

By using the $l_{1}$ norm as an approximation of the $l_{0}$ norm, we penalize representations which use more words than necessary. This sparsity constraint results in isolated peaks in $\mathbf{t}$ which can easily be detected using a peak detector such as is used for peak detection after the Hough transform. These detected peaks represent the centroids of the detected nuclei.

\section{Optimization}

In order to optimize the constrained problem in eq. (7) the problem is approximated by the following unconstrained optimization problem:

$$
\hat{\mathbf{u}}, \hat{\mathbf{t}}=\underset{\mathbf{t}, \mathbf{u} \in[0,1]^{m n}}{\arg \min }|\mathbf{t}|+\beta\langle\mathbf{u}, \mathbf{r}\rangle+\gamma\|\mathbf{u}-D \mathbf{t}\|_{2}^{2}
$$

Where $\gamma$ is a weighting parameter. Note that this only approximates the constraint $\mathbf{u}=$ $D$ t. Although there exists efficient techniques to enforce this constraint exactly, e.g. augmented Lagrangian or Bregman methods, we propose to use the approximation in eq. (8) instead. This allows the active contours to detect nuclei whose shape slightly deviates from the circular model or to detect partially overlapping nuclei. Given the convexity of eq. (8), this problem can be solved by iteratively optimizing for $\mathbf{t}$ and $\mathbf{u}$ independently, i.e.

$$
\begin{aligned}
& \hat{\mathbf{t}}=\underset{\mathbf{t}}{\arg \min }|\mathbf{t}|+\gamma\|\mathbf{u}-D \mathbf{t}\|_{2}^{2} \\
& \hat{\mathbf{u}}=\underset{\mathbf{u} \in[0,1]^{m n}}{\arg \min } \beta\langle\mathbf{u}, \mathbf{r}\rangle+\gamma\|\mathbf{u}-D \mathbf{t}\|_{2}^{2}
\end{aligned}
$$


The problem in eq. (9) is a typical $l_{1}-l_{2}$ optimization problem for which a variety of optimization methods have been proposed, e.g. FISTA, primal-dual methods, etc. [1618]. In the next section we evaluate several of these methods for the optimization of eq. (9). The problem in eq. (10) can be optimized by solving a set of Euler-Lagrange equations. For an optimal $\mathbf{u}$, the following optimality condition should be satisfied:

$$
\mathbf{u}=-2 \frac{\beta}{\gamma} \mathbf{r}+D \mathbf{t}
$$

The solution of eq. (11) is unconstrained, i.e. $\mathbf{u}$ does not have to lie in the interval $[0,1]^{m n}$. However minimizing eq. (10) is equivalent to minimizing a set of quadratic functions. So if $\mathbf{u}[i] \notin[0,1]$ then the constrained optimum is either 0 or 1 , since a quadratic function is monotonic in an interval which does not contain its extremum. So the constrained optimum is given by:

$$
\hat{\mathbf{u}}[i]=\max (\min (\mathbf{u}[i], 1), 0)
$$

\section{Results}

\subsection{Detection}

To validate the proposed method, a synthetic dataset is analyzed [19]. These synthetic images were proposed as a common benchmark for nuclei segmentation. The synthetic images show the same intrinsic properties of real microscopic images of cell nuclei: blurred nuclei, non uniform intensity in a nucleus, touching nuclei, non uniform background, etc. In Fig. 1(a) an example of such a synthetic image is shown. Fig. 1(b) shows the detection result using an edge based detection method [6]. The result of cellProfiler, i.e. an intensity based method [9], is shown Fig. 1(c), whereas Fig. 1(d) depicts nuclei detection using the proposed method. The proposed method shows more accurate results for the detection of isolated nuclei even if nuclei are clustered together.

To quantitatively validate the result, a dataset of 20 images, each containing 300 nuclei, were analyzed. The results are shown in Table 1 . The first row represents the results of an edge based method [6], whereas the next two rows correspond with two intensity based images $[9,10]$. Note that the proposed method, the last row, get the best results both for cell detection metrics, in the first 4 columns, as for the Dice coefficient, which is a metric for segmentation quality. Mark that lacking ground truth for individual nuclei, the Dice coefficient measures the similarity of all segmented nuclei compared with the ground truth for all nuclei instead of for individual nucleus.

In Fig. 2 an example of nuclei detection in a real microscopic image is shown. These microscopic image shows nuclei of peripheral blood mononuclear cells, captured with a PALM MicroBeam system. In Fig. 2.a shows the ground truth detection, which corresponds to manually annotations. Fig. 2.b and Fig. 2.c correspond to state of the art detection methods. Note that they both falsly detect nuclei at places where there is some smeared staining, i.e. when a nucleus ruptures it releases its staining, which results in bright smears. CellProfiler does not only suffer from false detections, but also merges touching nuclei. The proposed method does not suffer from false detection due to dye smears, while still being able to detect touching nuclei, as can be seen in Fig. 2.d. 


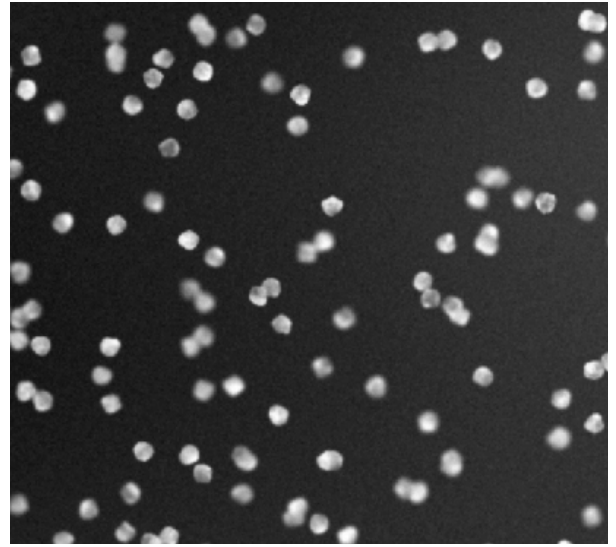

(a) Microscopic image

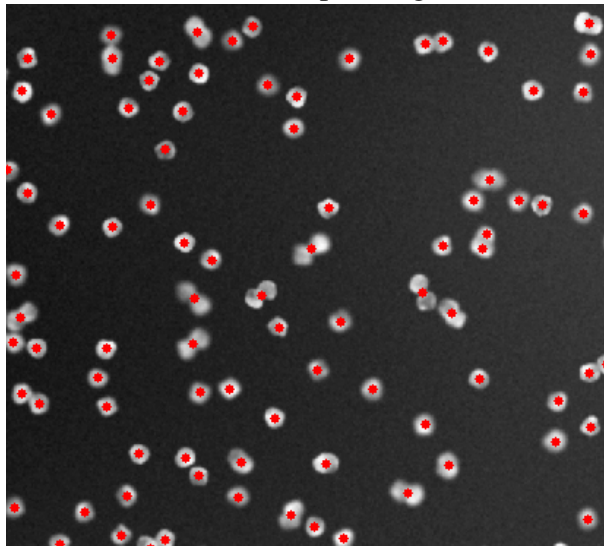

(c) cellProfiler

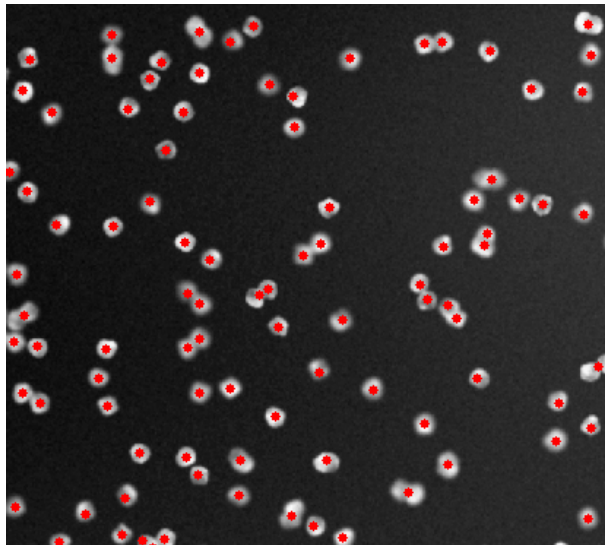

(b) edgeProp

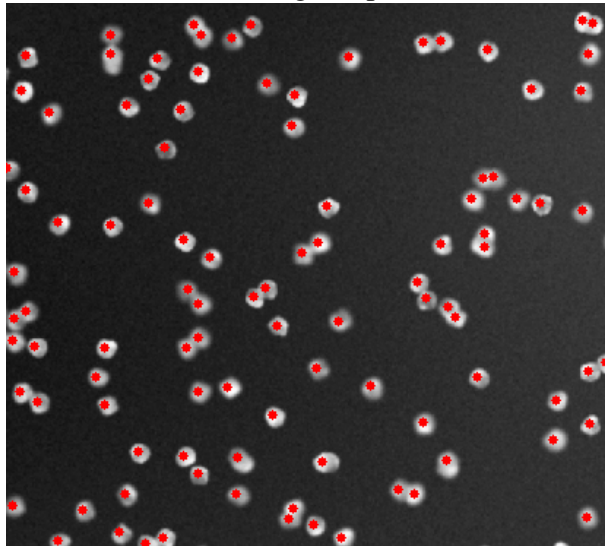

(d) Proposed method

Fig. 1. Example of cell detection using different methods on an image of the broad Bioimage Benchmark Collection 


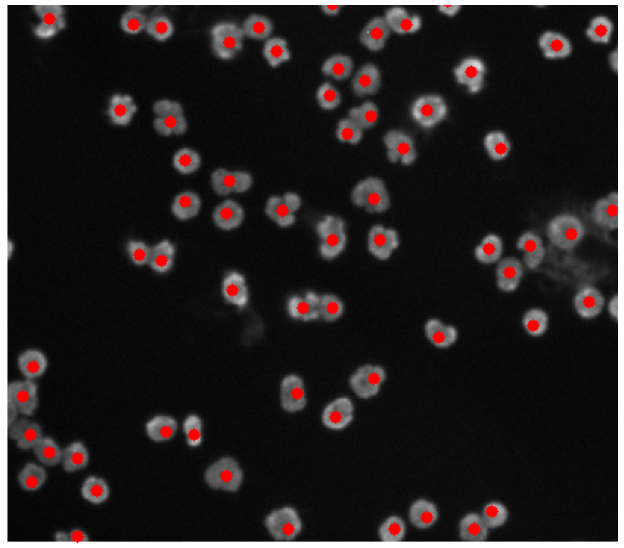

(a) ground truth

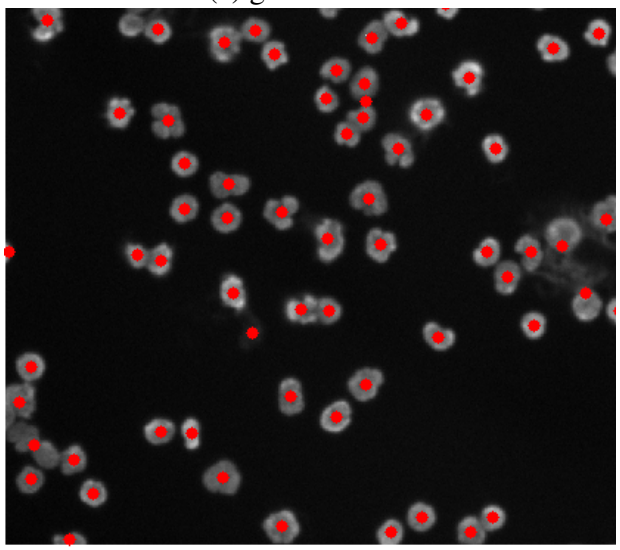

(c) cellProfiler

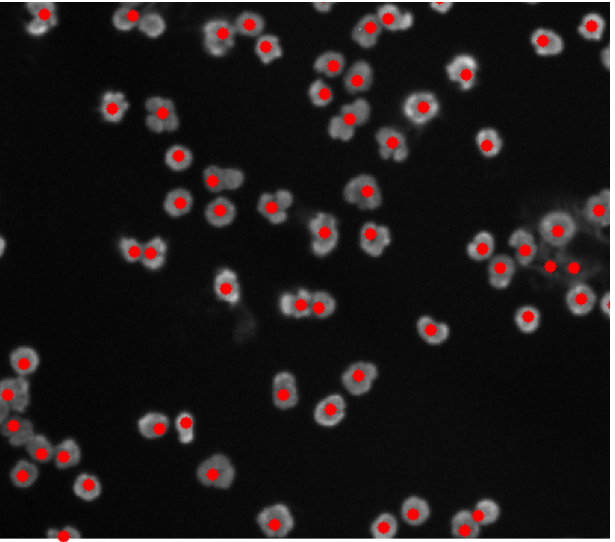

(b) edgeProp

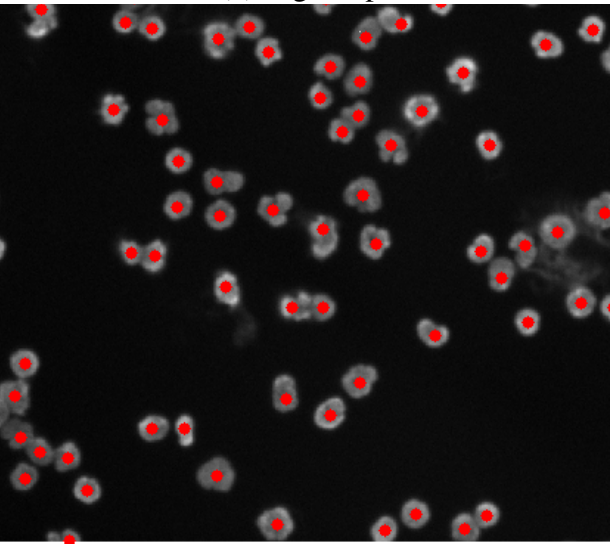

(d) Proposed method

Fig. 2. Example of cell detection using different methods on an image of the broad Bioimage Benchmark Collection 


\subsection{Convergence}

The optimal $\mathbf{u}$ and $\mathbf{t}$ are calculated by alternating optimizing eq. (8) for $\mathbf{u}$ and $\mathbf{t}$. This results in fast convergence as can be seen in Fig. 3, where the mean squared error of $\mathbf{u}$ and $\mathbf{t}$ are plotted after each iteration. Note that this error is in comparison with the optimal active contour, i.e. $\mathbf{u}$ and $\mathbf{t}$ after 10 iteration, and not to the ground truth segments. After 3 iterations an accurate approximation of $\mathbf{u}$ and $\mathbf{t}$ is already achieved.

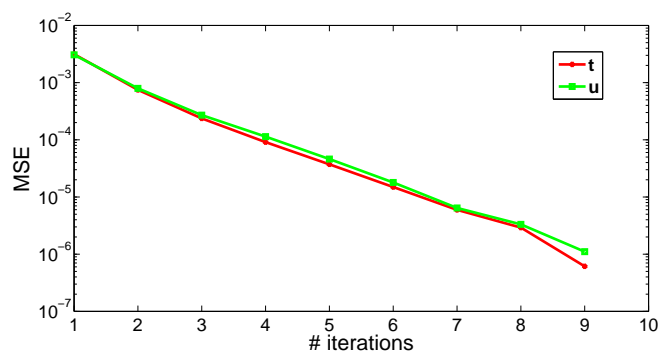

Fig. 3. Convergence of $\mathbf{u}$ and $\mathbf{t}$ in function of the number of iterations, the MSE is calculated between $\mathbf{u}$ and $\mathbf{t}$ and there optimal values, i.e. there values after convergence.

Solving the problem in eq. (9) requires to iteratively optimize an $l_{1}-l_{2}$ problem. For this type of problems several optimization methods have been proposed in literature [16-18]. In Table 2 we compare the performance of several of these methods: DALM[17], FISTA [18], L1LS [16], PALM [16] and SpaRSA[17]. The last column shows the computational time of a single iteration for the analysis of a $256 \times 256 \mathrm{im}$ age. These measurements where done using an Intel i7 Q720 1.6 GHz CPU with 4GB RAM. The L1LS optimizer needs significantly less iterations than the other tested methods, however a single iteration is much more time consuming. Therefore FISTA turns

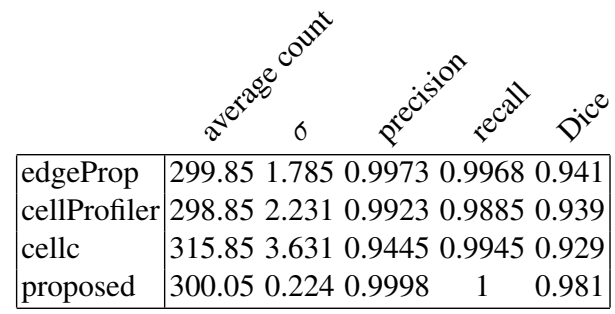

Table 1. A comparison of different cell nuclei detection and segmentation methods 
out to be the fastest method, with an average convergence after $6.10 \mathrm{~s}$. This results in a total processing time of $18.05 \mathrm{~s}$ for the complete segmentation of an image.

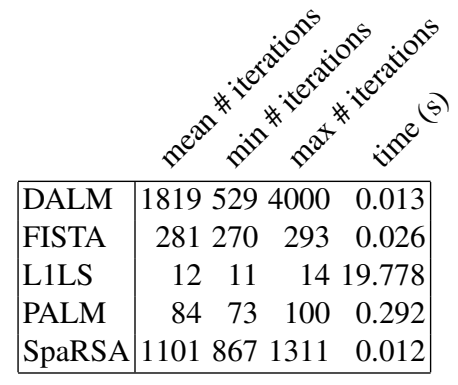

Table 2. A comparison of convergence of different optimization methods used for the inner optimization step, i.e. eq. (9)

\section{Conclusion}

This paper proposed a novel segmentation technique to detect and segment cell nuclei in fluorescent microscopic images. The method fits within the active contour framework has a convex energy function. The method uses prior knowledge about the shape of cell nuclei, which is done by representing the segmentation result using a dictionary. The proposed method was tested on a benchmark dataset, specifically proposed for this type of application. The method results in accurate nuclei detection and outperforms state of the art methods, e.g. precision and recalls of respectively 0.9998 and 1.

\section{Acknowledgment}

Jonas De Vylder is funded by the Institute for the Promotion of Innovation by Science and Technology in Flanders (IWT). The authors would like to thank the following institutes and researcher for sharing their codes and data: Tampere University of Technology for the use of CellC Cell Counting, Broad institute for providing both the benchmark dataset and the cellProfiler software and Allen Y. Yang (University of California, Berkeley) for the $l_{1}$ optimization benchmarking toolkit.

\section{References}

1. Keller, P.J., Schmidt, A.D., Wittbrodt, J., Stelzer, E.H.K.: Reconstruction of zebrafish early embryonic development by scanned light sheet microscopy. Science 322 (2008) 1065-1069

2. Chen, X.W., Zhou, X.B., Wong, S.T.C.: Automated segmentation, classification, and tracking of cancer cell nuclei in time-lapse microscopy. IEEE Transactions on Biomedical Engineering 53 (2006) 762-766 
3. Gladilin, E., Goetze, S., Mateos-Langerak, J., Van Driel, R., Eils, R., Rohr, K.: Shape normalization of $3 \mathrm{~d}$ cell nuclei using elastic spherical mapping. Journal of Microscopy-Oxford 231 (2008) 105-114

4. Hukkanen, J., Hategan, A., Sabo, E., Tabus, I.: Segmentation of cell nuclei from histological images by ellipse fitting. In: The 2010 European Signal Processing Conference. (2010)

5. Li, G., Liu, T., Nie, J., Guo, L., Chen, J., Zhu, J., Xia, W., Mara, A., Holley, S., Wong, S.T.C.: Segmentation of touching cell nuclei using gradient flow tracking. Journal of MicroscopyOxford 231 (2008) 47-58

6. De Vylder, J., Philips, W.: Computational efficient segmentation of cell nuclei in $2 \mathrm{~d}$ and $3 \mathrm{~d}$ fluorescent micrographs. In: proceedings of SPIE Photonics West : conference on Imaging, Manipulation and Analysis of Biomolecules, Cells, and Tissues. (2011)

7. Gudla, P.R., Nandy, K., Collins, J., Meaburn, K.J., Misteli, T., Lockett, S.J.: A highthroughput system for segmenting nuclei using multiscale techniques. Cytometry Part A 73A (2008) 451-466

8. Sezgin, M., Sankur, B.: Survey over image thresholding techniques and quantitative performance evaluation. Journal of Electronic Imaging 13 (2004) 146-168

9. Kamentsky, L., Jones, T.R., Fraser, A., Bray, M.A., Logan, D.J., Madden, K.L., Ljosa, V., Rueden, C., Eliceiri, K.W., Carpenter, A.E.: Improved structure, function and compatibility for cellprofiler: modular high-throughput image analysis software. Bioinformatics 27 (2011) 1179-1180

10. Selinummi, J., Seppala, J., Yli-Harja, O., Puhakka, J.A.: Software for quantification of labeled bacteria from digital microscope images by automated image analysis. Biotechniques 39 (2005) 859-863

11. Cloppet, F., Boucher, A.: Segmentation of overlapping/aggregating nuclei cells in biological images. In: 19th International Conference on Pattern Recognition, Vols 1-6. (2008) 789-792

12. Chan, T.F., Esedoglu, S., Nikolova, M.: Algorithms for finding global minimizers of image segmentation and denoising models. Siam Journal on Applied Mathematics 66 (2006) 16321648

13. Bresson, X., Chan, T.F.: Active contours based on chambolle's mean curvature motion. 2007 IEEE International Conference on Image Processing, Vols 1-7 (2007) 33-363371

14. Chan, T., Vese, L.: An active contour model without edges. Scale-Space Theories in Computer Vision 1682 (1999) 141-151

15. Baraniuk, R.G.: Compressive sensing. IEEE Signal Processing Magazine 24 (2007) 118-+

16. Kim, S.J., Koh, K., Lustig, M., Boyd, S., Gorinevsky, D.: An interior-point method for largescale 11-regularized least squares. IEEE Journal of Selected Topics in Signal Processing 1 (2007) 606-617

17. Yang, A.Y., Sastry, S.S., Ganesh, A., Yi, M.: Fast 11-minimization algorithms and an application in robust face recognition: A review. In: Image Processing (ICIP), 2010 17th IEEE International Conference on. (2010) 1849-1852

18. Zibulevsky, M., Elad, M.: L1-12 optimization in signal and image processing. IEEE Signal Processing Magazine 27 (2010) 76-88

19. Ruusuvuori, P., Lehmussola, A., Selinummi, J., Rajala, T., Huttunen, H., Yli-Harja, O.: set of synthetic images for validating cell image analysis. In: Proc. of the 16th European Signal Processing Conference (EUSIPCO-2008). (2008) 